\title{
INFLUENCE OF UAS PILOT COMMUNICATION AND EXECUTION DELAY ON CONTROLLER'S ACCEPTABILITY RATINGS OF UAS-ATC INTERACTIONS
}

\author{
Kim-Phuong L. Vu, Gregory Morales, Dan Chiappe, Thomas Z. Strybel, Center for Human Factors in \\ Advanced Aeronautics Technologies and California State University Long Beach, Long Beach, CA \\ Vernol Battiste, NASA Ames Research Center and San Jose State Foundation, Moffet Field, CA \\ Jay Shively, NASA Ames Research Center, Moffet Field, CA \\ Timothy J. Buker, SAIC, Washington, D.C.
}

\begin{abstract}
Successful integration of UAS in the NAS will require that UAS interactions with the air traffic management system be similar to interactions between manned aircraft and air traffic management. For example, UAS response times to ATCo clearances should be equivalent to those that are currently found to be acceptable with manned aircraft. Prior studies have examined communication delays with manned aircraft. Unfortunately, there is no analogous body of research for UAS. The goal of the present study was to determine how UAS pilot communication and execution delays affect ATCos' acceptability ratings of UAS pilot responses when the UAS is operating in the NAS. Eight radar-certified controllers managed traffic in a modified ZLA sector with one UAS flying in it. In separate scenarios, the UAS pilot verbal communication and execution delays were either short (1.5 s) or long (5 s) and either constant or variable. The ATCo acceptability of UAS pilot communication and execution delays were measured subjectively via post trial ratings. UAS verbal pilot communication delay, were rated as acceptable $92 \%$ of the time when the delay was short. This acceptability level decreased to $64 \%$ when the delay was long. UAS pilot execution delay had less of an influence on ATCo acceptability ratings in the present stimulation. Implications of these findings for UAS in the NAS integration are discussed.
\end{abstract}

\section{Introduction}

The FAA Modernization and Reform Act of 2012 calls for a plan to integrate unmanned aircraft systems (UAS) into the National Airspace System (NAS) by 2015 [1]. To achieve the goal of safe and non-disruptive flight in the NAS, however, UAS may be required to meet the same operational and certification standards as manned aircraft; they may have to "act and respond as manned aircraft do" (ICAO, 2011, p. 5) [2]. Research is required to precisely quantify what these standards should be [3]. However, although FAA regulations require that pilots respond promptly to air traffic controller (ATCo) commands and communications, they do not specify what an acceptable response delay actually is. The only stipulation is that delays must not compromise the safe separation of aircraft in the surrounding airspace. The present study examined how delays in verbal communications and command executions affected ATCo acceptability ratings of UAS and manned aircraft operating in a simulated NAS environment.

Currently, ATCos direct traffic by issuing verbal clearances to aircraft. Pilots read back the clearances, and maneuver their aircraft to carry out the instructions. ATCos are required to monitor pilot read backs, and to correct pilots if they make a mistake [4]. The end-to-end response time to ATCo clearances is referred to as the "measured response" (MR) $[5,6]$. This overall response time can be broken down into the following MR components: (1) Pilot verbal communication delay. The lag between the end of an ATCo clearance and the beginning of the pilot's readback. (2) Pilot execution delay. The lag between the end of the ATCo's command and when the pilot begins to initiate the maneuver (3) Aircraft response delay. The time between the pilot entering a command and the aircraft initiating action. (4) ATCo display delay. The time for the maneuver to be visible on the ATCo display once the aircraft has begun its maneuver. In the present study, we focus on the first two MR components-pilot verbal communication delay and pilot execution delay. We did not examine aircraft response time delays or 
display visibility delays because these cannot be manipulated by the version of the Multiple UASSimulator (MUSIM) ground control station (GCS) that we used for the study [7]. Specifically, the version of MUSIM used in the present simulation was not programmed to simulate a specific UAS; the aircraft responded immediately once pilots entered commands. Moreover, UAS maneuvers were visible on ATCo radar screens within a constant time period.

UAS differ from manned aircraft in several ways that are likely to affect their measured response. These include their size, speed, interfaces, and most critically, that UAS pilots are not co-located with their aircraft [8]. This fact can delay the response of both the pilot and UAS to ATCo commands, and it is important to determine what acceptable delays are.

Research has been conducted on some of the MR components for manned aircraft. Cardosi [9], for example, examined verbal exchanges between pilots and controllers from three en route sectors with the goal of identifying the time from the ATCo's initial transmission to the end of the pilot's response, and assessing the various components therein. She found a mean total communication time of approximately $10 \mathrm{sec}$, across three different types of clearances. Within this overall time, she found a mean pilot verbal delay of $3.31 \mathrm{sec}(\mathrm{SD}=4.80 \mathrm{sec})$ for maneuvers of traffic avoidance, a mean verbal delay of $2.68 \mathrm{sec}(\mathrm{SD}=4.60 \mathrm{sec})$ for turns not for traffic, and a mean verbal delay of $2.67 \mathrm{sec}(\mathrm{SD}=6.25 \mathrm{sec})$ for traffic advisories. Based on these results, however, one cannot determine what an acceptable pilot delay is. This is because of the large variability associated with each of these means, and the fact that the means are based on some pilot delays that were too long to be acceptable: On $12 \%$ of the communications, controllers had to repeat their commands and some of these were because the pilots initially failed to respond.

In a study conducted with recordings from TRACONs, Smith [10] found communication times between ATCos and pilots between $5.18 \mathrm{sec}$ and 8.62 sec depending on the type of maneuver. The delays between pilot and controller responses were also shorter than those identified by Cardosi [9], at about $1 \mathrm{sec}$. However, the latter includes both pilot verbal delays (i.e., when the ATCo initiates communication) and ATCo verbal delays (i.e., when the pilot initiates communication), making it difficult to determine ATCo acceptability of this range.

To summarize, based on the research on manned aircraft, it is difficult to quantify the acceptability of pilot delays in verbal responses. There is also little known about how long it takes pilots to begin executing commands and what is acceptable in this regard. We have begun conducting research to examine the acceptability of different MR components. Shively et al. [5], for example, had two ATCos issue 15 different clearances to pilots flying UAS using the MUSIM simulator. ATCos were not managing other traffic, and the pilots were not engaged in other UAS tasks. The pilots had to repeat the clearances, and execute the commands as quickly as possible. The different components of the MR were recorded, and the controllers rated the acceptability of the delays. The results revealed an average pilot communication delay of $2.5 \mathrm{sec}$, which is likely shorter than it would be under less ideal circumstances where pilots are engaged in other UAS tasks and are not expecting ATCo commands. Furthermore, the execution delay was found to vary considerably, ranging from $1.7 \mathrm{sec}$ to $7.63 \mathrm{sec}$. For some clearances, execution delays were found to be negatively correlated with ATCo acceptability ratings. That is, longer execution times were associated with lower acceptability ratings.

The present study furthers this research by manipulating the first two MR components and examining the consequences for ATCo acceptability ratings. In particular, we manipulated whether UAS pilot verbal responses were "short" or "long" by inserting a delay of either $1.5 \mathrm{sec}$ or $5 \mathrm{sec}$ to their verbal responses. We also manipulated whether the execution delays were short or long by adding a delay of either $1.5 \mathrm{sec}$ or $5 \mathrm{sec}$ prior to the beginning of their responses. The values for the pilot verbal delay were chosen based on the acceptability ratings collected in Shively et al. [5], where pilot verbal delays greater than $5 \mathrm{sec}$ were generally rated unacceptable by the ATCos. The current study also manipulated whether the delays were constant or variable. In the constant conditions, the scenarios either had all short or all long verbal delays, paired with either short or all long execution delays. In the variable conditions, however, some verbal delays were long and some short, and the same was the case with the execution delays. This allowed us to determine whether it is a delay per se that is 
problematic, or whether it is the predictability of the delays that is essential to their acceptability. It is possible, for example, that if the delays are constant, ATCos can develop strategies for accommodating them. This would be more difficult if the delays are unpredictable.

\section{Method}

\section{Participants}

Participants were 8 radar-certified Air Traffic Controllers, with a mean of 28 years of experience in military and civilian air traffic management. Six of the participants were recently retired. Participants were compensated $\$ 60$ per hour for their time.

\section{Design}

This simulation followed a 2 (Verbal Delay: Short or Long) x 2 (Execution Delay: Short or Long) x 2 (Predictability Constant or Variable) withinsubjects design. The additional delays were 1.5 seconds for the Short condition and 5 seconds for the Long condition. This generated four combinations of voice-execution delays: Voice-Short ExecutionShort, Voice-Short Execution-Long, Voice-Long Execution-Short, and Voice-Long ExecutionLong. The Constant delay condition used only one of the four delay combinations through an entire trial. The Varied delay condition switched between delay combinations during the trial to include two repetitions of each delay combination in random order.

\section{Apparatus}

The simulation was conducted in the Center for Human Factors in Advanced Aeronautics Technologies (CHAAT) at California State University, Long Beach. The simulation was run in the Multi Aircraft Control System (MACS) [11], which creates a medium-fidelity airspace environment. MACS simulated a DSR station for sector ZLA 20. All manned aircraft were flown by one pseudopilot using MACS in pseudopilot mode; the UAS was flown by a pseudopilot on the MUSIM GCS. In MUSIM, the pseudopilot controlled the altitude and point-to-point navigation of the UAS. The UAS (callsign PD-1) flew flight paths at altitudes between 10,000-16,000 ft. and at a constant cruise speed of 110 knots.

The ATCo, manned pseudopilot, and UAS pseudopilot communicated via push-to-talk headsets over a voice server. On MUSIM, voice transmissions were delayed by either $1.5 \mathrm{~s}$ or $5 \mathrm{~s}$, depending on the delay condition. The voice communication system also allowed for the production of "step-ons" in the event of simultaneous transmissions.

\section{Procedure}

The simulation was conducted over two days for each participant. On the morning of the first day participants completed consent forms, demographic questionnaires, and were briefed on simulation procedures. Following the briefing the controllers performed four 30-minute practice trials. Then, the ATCos performed two experimental blocks, each consisting of four, 40-minutes scenarios (one block in the afternoon of Day 1 and one block in the morning of Day 2). During the experimental trials, controllers managed all air traffic coming into their sector, which consisted of three LAX arrival streams and overflights. Controllers were instructed that arrival aircraft had priority and were required to leave the sector at an altitude no greater than 12,000 feet, and airspeed 250 knots. The controllers were told that their Center had a Letter of Agreement to accommodate PD-1's flight objectives while maintaining safe operations for all air traffic.

Beginning approximately 1-2 minutes from the start of the trial and every 4-5 minutes in alternating order, either "Mission Control" or the UAS would initiate a route modification request for PD-1. Mission Control was simulated on a separate computer located to the right of the controller's radar scope. This station would alert the controller through his headset and display a heading modification (for the UAS (e.g., "PD-1 proceed direct EDITS"). Mission control only requested the ATCo to direct the UAS to fixes. The ATCo had to issue PD-1 a new clearance to the fix. The UAS would request altitude clearances while flying to a directed fix. A total of 8 UAS changes were completed in each scenario, four initiated by the controller and four initiated by the UAS pseudopilot.

Voice and Execution delays were controlled at the UAS pseudopilot station. Voice software at this 
station automatically delayed transmission of the UAS pseudopilot's audio for the set 1.5 or 5 seconds before broadcasting it to the ATCo and manned pseudopilot. The UAS station only included a transmission delay, not a receiving delay. The ATCo and manned pseudopilots had no added receiving or transmission delays. After verbally responding to the clearance from the ATCo, the UAS pilot would activate a countdown timer of 1.5 or $5 \mathrm{~s}$ before beginning to execute the instruction. For the variable delay conditions, the voice and execution delays changed between clearances. Each voice-execution delay combination was presented twice in random order.

After each trial, controllers rated their situation awareness, workload, experience, and acceptability of interacting with the manned and UAS pseudopilots. Once all 8 experimental trials were completed, controllers answered post-simulation and debriefing questionnaires. Finally, the ATCos were interviewed during a debriefing session.

\section{Results}

The data reported in this paper represents only a subset of the data collected from the simulation. In the present paper, we report descriptive analyses of the two MR components and the acceptability rating data provided by ATCos for these components. Data from one participant was excluded from the analyses due to noncompliance with the experimental instructions.

The means and standard deviations for MR components 1 and 2, and their corresponding ATCo acceptability ratings are listed in Tables 1 and 2, respectively. Because the ATCo acceptability ratings were collected post-trial, the mean ratings are collapsed across the four variable scenarios.

\section{Table 1. Means and Standard Deviations for the MR Component 1, Verbal Response (s) and Acceptability Ratings.}

\begin{tabular}{|c|c|c|c|c|c|}
\hline \multirow[t]{2}{*}{ Condition } & \multirow[t]{2}{*}{ Pilot Role } & \multicolumn{2}{|c|}{$\begin{array}{l}\text { MR Component } 1 \\
\text { Communication Lag }\end{array}$} & \multicolumn{2}{|c|}{$\begin{array}{c}\text { Mean ATCo } \\
\text { Acceptability Rating }\end{array}$} \\
\hline & & Mean & Std. Dev & Mean & Std. Dev \\
\hline \multirow[t]{2}{*}{ Constant: VS-ES } & UAS & 2.07 & 0.50 & 5.14 & 0.69 \\
\hline & Manned* & 0.81 & 0.23 & 5.00 & 1.41 \\
\hline \multirow[t]{2}{*}{ Constant: VS-EL } & UAS & 2.12 & 0.27 & 4.86 & 1.57 \\
\hline & Manned* & 0.80 & 0.35 & 5.43 & 1.13 \\
\hline \multirow[t]{2}{*}{ Constant: VL-ES } & UAS & 5.52 & 0.32 & 4.00 & 1.41 \\
\hline & Manned* & 0.86 & 0.34 & 4.43 & 1.51 \\
\hline \multirow[t]{2}{*}{ Constant: VL-EL } & UAS & 5.43 & 0.25 & 4.43 & 1.39 \\
\hline & Manned* & 1.15 & 0.51 & 4.43 & 1.13 \\
\hline \multirow{2}{*}{$\begin{array}{l}\text { Variable } \\
\text { (averaged across the } \\
4 \text { variable scenarios) }\end{array}$} & UAS & 3.72 & 0.22 & 5.07 & 1.17 \\
\hline & Manned* & 0.94 & 0.52 & 5.43 & 1.02 \\
\hline
\end{tabular}

*For manned aircraft pseudopilots, only a small sample of pilot-ATCo interactions were examined 
Table 2. Means and Standard Deviations for the MR Component 2, Execution Response (s) and Acceptability Ratings.

\begin{tabular}{|c|c|c|c|c|c|}
\hline \multirow[t]{2}{*}{ Condition } & \multirow[t]{2}{*}{ Pilot Role } & \multicolumn{2}{|c|}{$\begin{array}{l}\text { MR Component } 2 \\
\text { Execution Lag }\end{array}$} & \multicolumn{2}{|c|}{$\begin{array}{c}\text { Mean ATCo } \\
\text { Acceptability Rating }\end{array}$} \\
\hline & & Mean & Std. Dev & Mean & Std. Dev \\
\hline \multirow[t]{2}{*}{ Constant: VS-ES } & UAS & 6.27 & 2.51 & 5.14 & 0.69 \\
\hline & Manned* & 3.24 & 2.21 & 5.00 & 1.83 \\
\hline \multirow[t]{2}{*}{ Constant: VS-EL } & UAS & 10.57 & 1.32 & 5.00 & 1.00 \\
\hline & Manned* & 4.79 & 1.47 & 5.43 & 0.98 \\
\hline \multirow[t]{2}{*}{ Constant: VL-ES } & UAS & 7.17 & 1.43 & 4.43 & 1.90 \\
\hline & Manned* & 5.56 & 3.48 & 5.29 & 0.95 \\
\hline \multirow[t]{2}{*}{ Constant: VL-EL } & UAS & 9.98 & 2.34 & 4.43 & 1.13 \\
\hline & Manned* & 4.97 & 2.45 & 5.29 & 1.38 \\
\hline \multirow{2}{*}{$\begin{array}{l}\text { Variable } \\
\text { (averaged across the } \\
4 \text { variable scenarios) }\end{array}$} & UAS & 8.98 & 1.24 & 5.18 & 1.00 \\
\hline & Manned* & 5.58 & 2.43 & 5.71 & 0.78 \\
\hline
\end{tabular}

*For manned aircraft pseudopilots, only a small sample of pilot-ATCo interactions were examined

\section{MR 1: Pilot Verbal Communication Delay}

Mean MR-1 component times were computed for each participant in each condition. For the variable condition, we extracted the data for the specific voice and execution delay implemented in the pilot-ATCo interaction. Mean response time for MR1 was submitted to a 2 (Verbal delay: short vs. long) $\mathrm{x} 2$ (Execution delay: short vs. long) $\mathrm{x} 2$ (Predictability: constant vs. variable) x 2 (Pilot Role: UAS vs. manned) mixed ANOVA. Pilot Role was the only between-subjects variable.

The main effect for Verbal Delay was significant, $F(1,12)=2004, p<.001$. As expected, the time interval between the end of an ATCo verbal clearance and the pilot's read back was shorter in the conditions with the short UAS verbal delays $(M=$ $1.41 \mathrm{sec})$ compared with long delays $(M=3.27 \mathrm{sec})$. In addition, there was a significant main effect of Pilot Role, $F(1,12)=959, p<.001$, where the verbal communication lag was shorter for manned aircraft $(M=0.92 \mathrm{sec})$ than the UAS $(M=3.75 \mathrm{sec})$. Again, this finding is expected because only the UAS transmissions were delayed. These two main effects were qualified by a significant two-way interaction of both variables, $F(1,12)=1502, p<.001$. As shown in Figure 1, there was a large difference in UAS verbal communication delay as a function of the verbal delays that was not evident with manned aircraft.

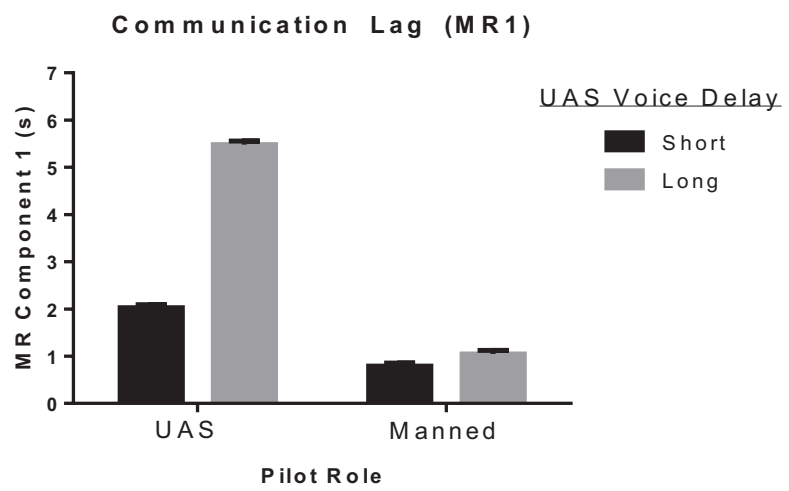

Figure 1. Verbal Response Time (MR-1) as a Function of UAS Verbal Delay $x$ Pilot Role 


\section{MR 2: Pilot Execution Delay}

The same ANOVA was run for the MR-2 component. The main effects for Verbal Delay, $F(1,12)=5.5, p=.03$, and Pilot Role, $F(1,12)=23.4$, $p<.001$, were significant. The time from when the ATCo completed issuing the clearance to when the pilot started to execute the clearance was shorter in the conditions with the short UAS verbal delay $(M=$ $6.69 \mathrm{sec})$ than long delay $(M=7.16 \mathrm{sec})$. In addition, the execution time was shorter for manned aircraft $(M=5.11 \mathrm{sec})$ than the UAS $(M=8.74 \mathrm{sec})$.

For MR 2, the main effects of Execution Delay, $F(1,12)=56.3, p<.001$, and Predictability, $F(1,12)=$ $4.7, p=.05$, were also significant. Pilots initiated the execution of a clearance faster for short UASexecution delays $(M=6.21 \mathrm{sec})$ than long delays $(M$ $=7.64 \mathrm{sec})$. The execution response was also faster when the delays were constant $(M=6.57 \mathrm{sec})$ than variable $(M=7.64 \mathrm{sec})$.

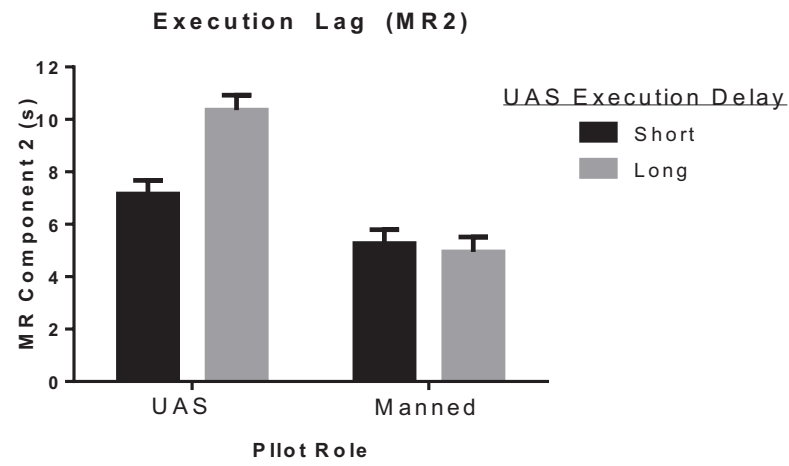

Figure 2. Execution Response Time (MR 2) as a function of UAS Execution Delay x Pilot Role

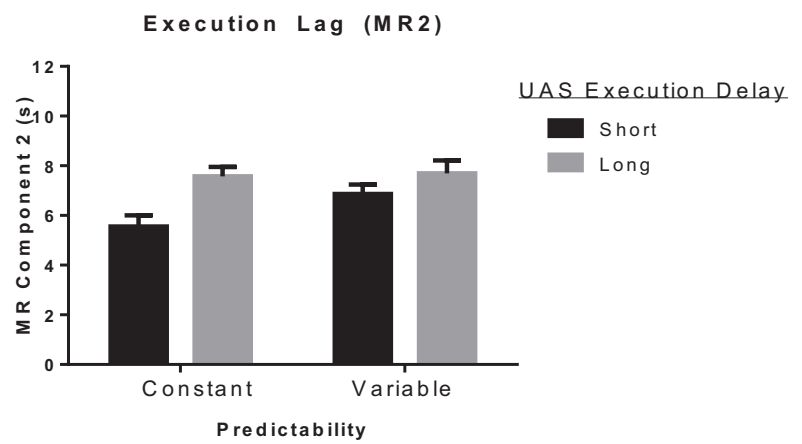

Figure 3. Execution Response Time (MR 2) as a function of UAS Execution Delay and Predictability
Execution delay entered into two, two-way interactions; one with Pilot Role, $F(1,12)=83.6, p<$ .001 , and the other with Predictability, $F(1,12)=6.2$, $p=.03$. As shown in Figure 2, there was a large difference in UAS pilots' MR 2 response times as a function of the induced pilot execution delays that was not evident with pilots of manned aircraft. Figure 3 illustrates that the difference between the short and long execution delay was more evident when the delay is constant than when it is variable.

\section{ATCo Acceptability Ratings}

Because the ATCo acceptability ratings were collected post-trial, we could only examine the effects of the verbal and execution delay variables independently for the constant delay conditions. Acceptability was rated on a 1 (not at all acceptable) to 7 (very acceptable) scale.

Because the ATCos rated both the acceptability of pseudopilots flying UAS and manned aircraft, a 2 (Verbal delay: short vs. long) x 2 (Execution delay: short vs. long) $\mathrm{x} 2$ (Pilot Type: UAS vs. conventional) repeated measures ANOVA was performed. No effects were significant. In general, the overall communication lag and execution lag in all conditions were judged to be acceptable (all means greater than or equal to the midpoint, i.e., 4.0, of the acceptability rating scale). To examine the effects of predictability, a second ANOVA was performed on acceptability ratings with Predictability (constant vs. variable) as a factor. Again, this analysis yielded no significant effects.

Correlations were computed between ATCo acceptability rating and MR component for the constant verbal and execution delay conditions separately for UAS and manned aircraft. For UAS, the correlation between acceptability ratings and MR 1 was marginally significant, $r=-0.35, p=.07$ but the correlation between acceptability and MR 2 was nonsignificant, $r=-0.19$. Shorter verbal delays were associated with higher acceptability ratings. For manned aircraft, the correlations were nonsignificant for MR 1, $r=-0.29$ and MR 2, $r=-0.03$. Note also that acceptability ratings of MR 1 and MR 2 were significantly correlated, $r=0.74, p<.001$, suggesting that ATCos had difficulty separating the contribution of each measured response to acceptability. 


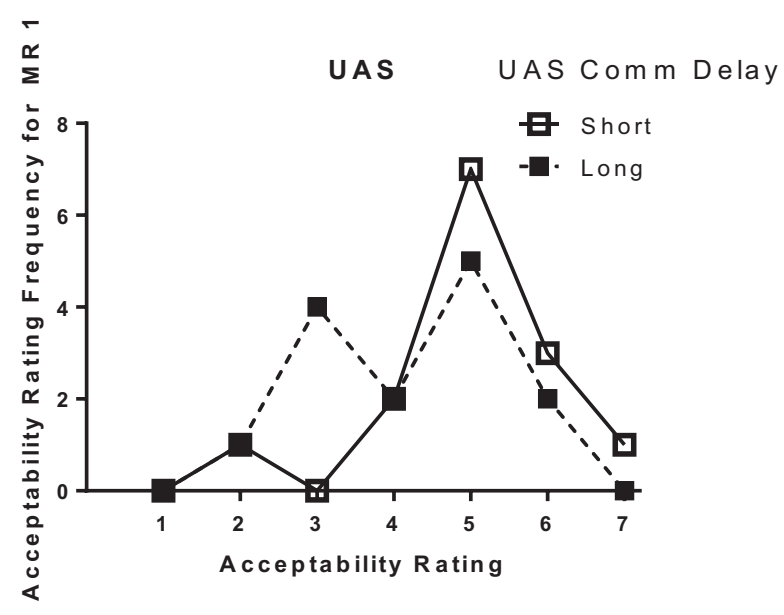

Figure 4. Distribution of UAS MR-1 Acceptability Ratings for Short and Long Communication Delays

Figure 4 shows the distribution of acceptability ratings of short and long delays for UAS. For short delays, $92 \%$ of the ratings were acceptable; for long delays, $64 \%$ of the ratings were acceptable. Figure 5 shows these ratings for manned aircraft. Again, the majority of the ratings were in the acceptable range: $92 \%$ of the ratings were acceptable for short UAScommunication delays, and $71 \%$ were acceptable for long UAS-communication delays.

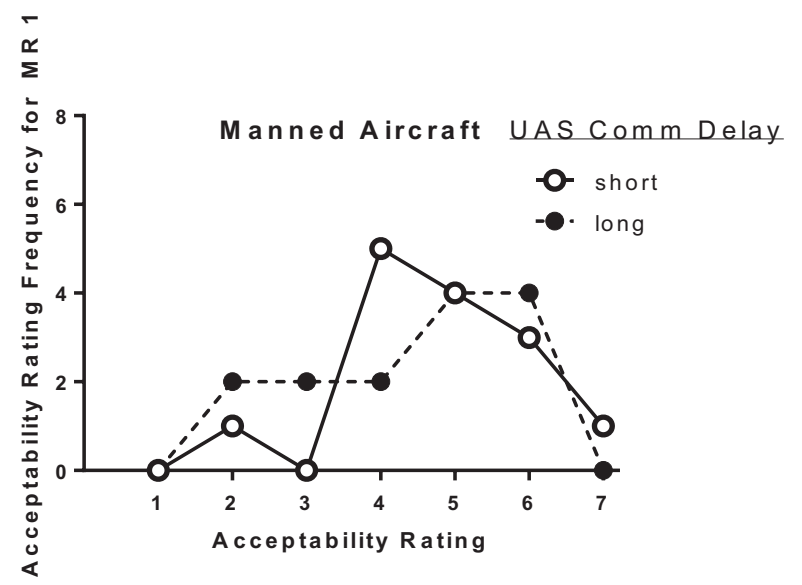

Figure 5. Distribution of Manned Aircraft MR-1 Acceptability Ratings for Short and Long UAS Communication Delays

\section{Discussion}

The present study examined the effects of UAS verbal and execution delays on ATCo acceptability ratings of a UAS operating in the NAS. We were able to extract the Pilot verbal communication response latency (MR 1 component) and execution response latency (MR component 2) in the simulation for UAS and manned aircraft.

For the pilot verbal communication delays, the mean response latency for manned aircraft was $0.78 \mathrm{~s}$ for the short delay condition and $1.06 \mathrm{~s}$ for the long delay condition. Thus, the induced UAS pilot verbal communication delay also lengthened the communication responses of manned aircraft. This lengthening in communication lag for the manned aircraft could be a result of step-ons that were associated with the long UAS verbal delays, because the number of times the UAS transmissions stepped on the transmissions of ATCos or manned aircraft increased in the long verbal-delay conditions.

Thus, inclusion of the UAS with long pilot verbal communication delays can push the manned aircraft pilots' verbal communication lag into unacceptable regions, even though the latencies for manned aircraft were 3-5 times faster than UAS latencies. Nevertheless, in the present simulation, the responses of manned aircraft were rated unacceptable on $29 \%$ of the long delay simulation trials, and were rated unacceptable on $8 \%$ of the short delay simulation trials. For long delays the percentage of unacceptable ratings is larger than the $12 \%$ of communications that ATCos had to repeat in the study conducted by Cardosi [9].

The UAS pilots' verbal communication delay was $2.03 \mathrm{~s}$ in the short verbal delay condition and 5.53 in the long verbal delay condition. Consequently, only $64 \%$ of the acceptability ratings were in the acceptable range for long delays. However, the UAS execution delay was not related to ATCo acceptability ratings for either manned aircraft or UAS. One possible reason for this lack of an effect of execution delay was the slow speed (PD-1's speed was only 110 knots) of the UAS. During the debriefing session, the ATCos indicated that they did not really pay attention to the execution time because the slow speed of UAS meant that the execution delay did not disrupt other traffic. The ATCos indicated that the execution delay would have been a bigger factor if the UAS was faster. For manned aircraft, all but one execution time was rated as acceptable (4 or higher) with the outlier given a rating of 3. As such, there was little variability in 
acceptability ratings for the execution times for the conventional pilots.

In conclusion, our simulation showed that an average UAS pilot verbal communication delay of 2 sec was acceptable nearly $100 \%$ of the time, but an average delay of $5.5 \mathrm{sec}$ was only acceptable slightly more than half of the time. Note also ATCos rated a manned aircraft delay as unacceptable on about $30 \%$ of the transmissions in the long delay condition, despite the fact that this value is much less than the verbal delay of the UAS. The additional tolerance in pilot verbal delay shown by controllers is likely a result of the ATCos' accommodations for the one UAS in their sector. Moreover, the fact that acceptability ratings of communication and execution delays were highly correlated suggests that ATCos may have had difficulty determining the unique contribution of each MR component to acceptability.

\section{Acknowledgements}

This project was supported by NASA cooperative agreement NNX12AH23A, NASA UAS in the NAS (Jay Shively and Walter Johnson, Technical Monitors).

\section{References}

1. 112th Congress (2012). FAA Modernization and Reform Act of 2012. Retrieved from http://www.gpo.gov/fdsys/pkg/CRPT112hrpt381/pdf/CRPT-112hrpt381.pdf.

2. ICAO. (2011). Unmanned Aircraft Systems (UAS) (ICAO Cir 328). Montreal, Canada: International Civil Aviation Organization.

3. Dillingham, G. L. (2013). Unmanned aircraft systems: Continued coordination, operational data, and performance standards needed to guide research and development. Washington, DC: Government Accountability Office.

4. Hopkin, V. D. (1995). Human Factors in Air Traffic Control. Taylor \& Francis.

5. Shively, R. J., Vu, K.-P. L., \& Buker, T. J. (2013, October). Unmanned aircraft system response to air traffic control clearances: Measured
Response. Accepted for presentation at the Human Factors and Ergonomics Society International Annual Meeting. San Diego, CA.

6. Ziccardi, J., Roberts, Z., O'Connor, R., Rorie, C., Morales, G., Battiste, V., Strybel, T. Z., Chiappe, D., Vu, K.-P. L., \& Shively, J. (2013). Measuring UAS pilot responses to common air traffic clearances. In S. Yamamoto (Ed.): HIMI/HCII 2013, Part II, Lecture Notes in Computer Science, 8017, 606-612.

7. Fern, L., \& Shively, J. (2011). Designing airspace displays to support rapid immersion for UAS handoffs. In Proceedings of the Human Factors and Ergonomics Society 55th Annual Meeting (pp. 81-85). Santa Monica, CA: Human Factors and Ergonomics Society.

8. Williams, K. W. (2007). An Assessment of Pilot Control Interfaces for Unmanned Aircraft. (Report no. DOT/FAA/AM-07/8). Washington, DC: U.S. Department of Transportation, Federal Aviation Administration,Office of Aerospace Medicine.

9. Cardosi, K. M. (1993). Time Required for Transmission of Time-Critical Air Traffic Control Messages in an En Route Environment. The International Journal of Aviation Psychology, 3(4), 303-314.

10. Smith, E. C. (2008). Analysis of controller-pilot communication performance in Area Navigation (RNAV) and conventional arrival operations. Digital Avionics Systems Conference, 2008. DASC 2008. IEEE/AIAA $27^{\text {th }}$, vol., no., pp.4.A.61-4.A6-12, 26-30 OCT. 2008.

11. Prevot, T. (2002). Exploring the many perspectives of distributed air traffic management: The Multi Aircraft Control System: MACS. International Conference on HumanComputer Interaction in Aeronautics, HCI-Aero 2002, 23-25.

32nd Digital Avionics Systems Conference October 6-10, 2013 